\section{Ovarian Papillary Cystadenocarcinoma in Sub Saharan Africa: A Case Report}

\section{Abstract}

Introduction: Ovarian cancer is the fifth most common cancer of women; it accounts for $3.8 \%$ of all neoplasms. It concerns women over 45 years old and its revelation is late. Ovarian papillary cystadenocarcinoma is the most frequent histological form in $75 \%$ of cases. Its prognosis is bleak because of its late diagnosis. The purpose of this observation is to discuss its diagnostic and therapeutic approach in a context of limited resources.

Patient and methods: It is a case study realized at Bobo-Dioulasso's Teaching Hospital Center (Burkina Faso).

Results: We report here the case of an ovarian papillary cystadenocarcinoma in a 28 year old patient, revealed by a pelvic tumor, without alteration of the general state.

Conclusion: The malignant ovarian tumor is possible before 30 years; abdominopelvic computed tomography should supplement abdominopelvic ultrasound in the event of diagnosis of ovarian tumor to better define the nature of lesions and their degree of invasion and allow better planning of treatment.

Keywords: Ovarian tumor; Diagnosis; Treatment

\section{Kambire $\mathrm{JL}^{1 *}$, Sanon $\mathrm{BG}^{2}$, Zare $\mathrm{C}^{2}$ and Kambou $\mathrm{T}^{2}$}

\author{
1 Ouahigouya's Teaching Hospital Center, \\ Burkina Faso \\ 2 Bobo-Dioulasso's Teaching Hospital \\ Center, Burkina Faso
}

\section{*Corresponding author: \\ Dr. Jean Luc Kambire}

jeanluckambire@yahoo.fr

Assistant of General Surgery, Ouahigouya's Teaching Hospital Center, Burkina Faso.

Tel: $(+226) 70338914$

Citation: Kambire JL, Sanon BG, Zare

C, Kambou T (2017) Ovarian Papillary

Cystadenocarcinoma in Sub Saharan Africa: A Case Report. J Clin Epigenet. Vol. 3 No. 3:23

Received: July 17, 2017; Accepted: July 25, 2017; Published: July 31, 2017

\section{Introduction}

Ovarian cancer is the fifth cancer of women; it accounts for $3.8 \%$ of all neoplasms. It concerns women over 45 years old and its revelation is late. Ovarian papillary cystadenocarcinoma is the most frequent histological form in $75 \%$ of cases. Its prognosis is bleak because of its late diagnosis. The purpose of this observation is to discuss its diagnostic and therapeutic approach in a context of limited resources.

\section{Clinical Observation}

This patient was a 28-year-old woman who has been received a general surgery consultation for an abdominal tumor that had been evolving for 18 months without other associated signs. On examination, the general condition was preserved; there was an oval, firm, not very sensitive tumefaction, localized to the left iliac fossa. The rest of the abdomen was slightly increased in volume, painless, mat at the percussion with hydroaeric noises. An abdominal-pelvic ultrasound showed a left ovarian tumor; computed tomography was not available. A laparotomy was indicated after completion of the operability assessment. In peroperative, a probable peritoneal carcinosis of a left ovarian tumor was detected with invasion of the cecum, the great omentum and the peritoneum. A left ovariectomy, an epiploic and peritoneal biopsy were performed. The surgery was complicated $48 \mathrm{~h}$ later with a hyperthermia and a coma and the patient's death occurred on the $4^{\text {th }}$ postoperative day. The histological result concluded later to a papillary cystadenocarcinoma of the ovary with metastases of the peritoneum and omentum.

\section{Comments}

Ovarian cancer is the fifth cancer of women; it represents $3.8 \%$ of neoplasias, or 4,500 new cases of ovarian cancers in France. Several theories explaining ovarian carcinogenesis have succeeded one another in time: the historical theory of incessant ovulation supported by Fathala since 1971; hormonal theory implicating hyperandrogenism and luteal insufficiency; the theory of chronic inflammation; the tubal origin of ovarian cancers in progress since 2000 explains only $40 \%$ to $50 \%$ of ovarian cancers; an integrated model is currently recognized: The serous tumors of the ovary, fallopian tube tumors and peritoneal tumors are derived from the tubal pavilion and are the result of gene mutations that result in low-grade or high-grade ovarian tumors [1,2]. 
Thus, several risk factors are recognized for ovarian cancers: Age, genetic factors and hormonal factors. No genetic investigation was done in our patient; it could have demonstrated the presence of BRCA1 and BRCA2 genes or a Lynch syndrome, pathognomonic of genetic predisposition [3]. If the age of ovarian cancer oscillates between 40 and 65 years, our patient was only 28 years old. Ovarian cancer can remain silent for a long time, making clinical diagnosis difficult. The clinical symptomatology is polymorphous: It can be manifested by abdomino-pelvic pain, abdominal meteorism, and metrorrhagia; digestive or urinary disorders may reveal the disease. At a later stage, the tumor can be revealed by a pelvic mass or a tumor-ascitic syndrome with an alteration of the general state [4]. In our observation, the patient presented a pelvic mass without alteration of her general condition. Imaging is fundamental in the diagnostic process; Abdomino-pelvic ultrasound is performed in first intention as was the case in our observation; if it allows the diagnosis of ovarian tumor, the computed tomography is more efficient and evaluates the ratios of the tumor with the neighboring organs. Magnetic resonance imaging assesses peritoneal sites, presence of pelvic or aortic adenomegalies, involvement of the digestive tract, and presence of visceral metastases [4]. These explorations could not be done in our patient, because of their geographical and financial inaccessibility. No tumor marker assay was performed.

Our patient was operated at the end of a biological check-up. In peroperative, a probable peritoneal carcinosis of a left ovarian tumor with invasion of the cecum, the great omentum and the peritoneum was detected. A left ovariectomy of approximately $900 \mathrm{~g}$, an epiploic and peritoneal biopsy were performed. The standard operation consisting of a complete surgical

\section{References}

1 Pick JM, van Diest PJ, Zweemer RP, Jansen JW, Poort-Keesom RJ, et al. (2001) Dysplastic changes in prophylactically removed Fallopian tubes of women predisposed to developing ovarian cancer. J Pathol 195: 451-456.

2 Chêne G (2001) Ovarian carcinogenesis: recent and past hypotheses. Gynecologie Obstetrique et Fertilite 39: 216-223. cytoreduction was not performed. An iterative laparotomy was considered after interval chemotherapy. But the patient succumbed on the $4^{\text {th }}$ postoperative day. The histological result later concludes with a papillary cystadenocarcinoma of the ovary with metastases of the peritoneum and omentum. Ovarian cancer is known for its poor prognosis due to its silent evolution and the lack of preventive measures applicable to the female population even though ongoing clinical trials with the realization of a systematic ultrasound and the dosage of CA 125 , give hope, the radiological explorations must be available in order to allow a better evaluation of the lesions and better planification of the treatment. Indeed, a better evaluation of the lesions by realization of the tomography and of the magnetic resonance imaging in our patient, would have allowed us to adopt the inverse sequence [5].

\section{Conclusion}

Ovarian cancer is one of the most deadly; while waiting for effective preventive measures to be validated, means of radiological investigations must be available in order to allow better management of the tumoral lesions that are developing more and more in sub-Saharan Africa.

\section{Conflict of Interest}

The authors do not declare any conflicts of interest.

\section{Contribution of Authors}

All the authors mentioned contributed to the elaboration of this manuscript.

3 Pavelka JC, Li AJ, Karlan BY (2007) Hereditary ovarian cancerassessing risk and prevention strategies. Obstet Gynecol Clin North Am 34: 651-665.

4 Bats AS, Barranger E (2008) Mise au point sur les cancers of the ovaries. Journal de Gynécologie Obstétrique et Biologie de la Reproduction 37: 64-68.

5 Vergote I, Tropé CG, Amant F, Kristensen GB, Ehlen T, et al. (2010) Neoadjuvant chemotherapy or primary surgery in stage IIIc or IV ovarian cancer. N Engl J Med 363: 943-953. 